\title{
Prevalence and characterization of methicillin-resistant Staphylococcus aureus carrying mecA or mec $C$ and methicillin-susceptible Staphylococcus aureus in dairy sheep farms in central Italy
}

\author{
G. Giacinti, ${ }^{*}$ V. Carfora, $\uparrow$ A. Caprioli, $\uparrow$ D. Sagrafoli, ${ }^{*}$ N. Marri, ${ }^{*}$ G. Giangolini, ${ }^{*}$ R. Amoruso, $\dagger$ M. Iurescia, $\dagger$ \\ F. Stravino, $\uparrow$ S. Dottarelli, $\uparrow$ F. Feltrin, $†$ A. Franco, $\uparrow$ S. Amatiste, ${ }^{*}$ and A. Battisti ${ }^{1}$ \\ ${ }^{*}$ National Reference Centre for Ovine and Caprine Milk and Dairy Products Quality (CRELDOC), and \\ †General Diagnostic Department, National Reference Laboratory for Antimicrobial Resistance, Istituto Zooprofilattico Sperimentale del Lazio e \\ della Toscana “M. Aleandri” Via Appia Nuova 1411, 00178 Rome, Italy
}

\begin{abstract}
Between January and May 2012, a total of 286 bulk tank milk samples from dairy sheep farms located in central Italy were tested for the presence of Staphylococcus aureus. One hundred fifty-three samples were positive for $S$. aureus (53.5\%), with an average count of $2.53 \log \mathrm{cfu} / \mathrm{mL}$. A total of 679 S. aureus colonies were screened for methicillin resistance by the cefoxitin disk diffusion test, and 104 selected cefoxitin-susceptible isolates were also tested for their susceptibility to other antimicrobials representative of the most relevant classes active against Staphylococcus spp. by using the Kirby-Bauer disk diffusion method. Two methicillinresistant Staphylococcus aureus (MRSA) isolates, carrying respectively the mecA and the $m e c C$ genes, were detected in 2 samples from 2 different farms (prevalence $0.7 \%$ ). The mecA-positive MRSA isolate was blaZ positive, belonged to spa type t127, sequence type (ST)1, clonal complex (CC)1, carried a staphylococcal cassette chromosome mec (SCCmec) type IVa, and was phenotypically resistant to all the $\beta$-lactams tested and to erythromycin, streptomycin, kanamycin, and tetracycline. The mecC-positive MRSA isolate was negative for the chromosomally or plasmid-associated blaZ gene but positive for the blaZ allotype associated with SCCmec XI (blaZ-SCCmecXI), belonged to spa type 843, $\mathrm{ST}(\mathrm{CC}) 130$, carried a SCCmec type XI, and was resistant only to $\beta$-lactams. Both MRSA were negative for the presence of specific immune-evasion and virulence genes such as those coding for the Panton-Valentine leucocidin, the toxic shock syndrome toxin 1, and the immune evasion cluster genes. Regarding the presence of the major $S$. aureus enterotoxin genes, the mecC-
\end{abstract}

Received March 27, 2017.

Accepted June 17, 2017.

${ }^{1}$ Corresponding author: antonio.battisti@izslt.it positive MRSA tested negative, whereas the ST (CC)1 $m e c A$-positive MRSA harbored the seh gene. Among the 104 methicillin-susceptible $S$. aureus isolates examined for antimicrobial susceptibility, 63 (60.58\%) were susceptible to all the antimicrobials tested, and $41(39.42 \%)$ were resistant to at least 1 antimicrobial. In particular, 23 isolates $(22.12 \%)$ were resistant to tetracycline, $16(15.38 \%)$ to sulfonomides, $14(13.46 \%)$ to trimethoprim and sulfamethoxazole, and $9(8.65 \%)$ to ampicillin, whereas only 1 isolate was resistant to both fluoroquinolones and aminoglycosides. The high prevalence of $S$. aureus found in bulk tank milk samples and the isolation of MRSA, although at a low prevalence, underlines the importance of adopting control measures against $S$. aureus in dairy sheep farms to minimize the risks for animal and public health. Moreover, this study represents the first report of mec $C$-positive MRSA isolation in Italy and would confirm that, among livestock animals, sheep might act as a mecC-MRSA reservoir. Although this lineage seems to be rare in dairy sheep $(0.35 \%$ of farms tested), because mec $C$-positive MRSA are difficult to detect by diagnostic routine methods employed for mecA-positive livestock-associated MRSA, diagnostic laboratories should be aware of the importance of searching for the mecC gene in all the $m e c A$-negative $S$. aureus isolates displaying resistance to oxacillin, cefoxitin, or both.

Key words: sheep milk, Staphylococcus aureus, methicillin-resistant Staphylococcus aureus, mecA/mecC

\section{INTRODUCTION}

Staphylococcus aureus is involved in a wide variety of diseases in humans and animals and its pathogenicity is mainly related to a combination of genetic characteristics mediating virulence, invasive capacity, immune evasion, and antibiotic resistance (Chua et al., 2014). Staphylococcus aureus is a common cause of IMI in dairy ruminants, causing both clinical and subclinical 
forms accompanied with relevant economic losses due to reduced milk production and quality (Bergonier et al., 2003).

In the last years, the emergence of livestock-associated (LA) methicillin-resistant Staphylococcus aureus (MRSA) has been increasingly reported worldwide, with a raising concern for the risks of zoonotic transmission, especially for people with occupational livestock exposure (Hanselman et al., 2006; Vanderhaeghen et al., 2010; Fessler et al., 2012; Guardabassi et al., 2013), but also for the possible introduction of these strains in the community through the food chain (Kluytmans, 2010). Clonal complex (CC)398 is the most prevalent LA-MRSA lineage in Europe, although in Italy other major LA-MRSA lineages, such as CC1 and CC97, have spread and have also been found to colonize and cause infections in livestock (Alba et al., 2015; Feltrin et al., 2015; Luini et al., 2015; Carfora et al., 2016). In the last decade, MRSA clones with a divergent mecA homolog, named mecC (formerly mecALGA251), have been detected in different animal species and human beings in different European countries, with isolates mainly belonging to CC130, CC1943, and CC425 (GarcíaÁlvarez et al., 2011; Paterson et al., 2014; Angen et al., 2017). Zoonotic transmission of mec $C$-MRSA has been previously reported (Harrison et al., 2013; Petersen et al., 2013), although data on the prevalence, animal reservoir, and epidemiology of mecC-MRSA are still limited (Harrison et al., 2013; Petersen et al., 2013).

In recent years, our research group has been investigating the presence and the characteristics of $S$. aureus, particularly MRSA, from sheep dairy products and sheep farms of central Italy (Carfora et al., 2015; Carfora et al., 2016), an area where the milk and cheese manufacturing industry is well developed and the consumption of raw milk dairy products of ovine origin is quite popular.

In this paper we report data on the prevalence of $S$. aureus in the bulk tank milk (BTM) samples collected from dairy sheep farms located in central Italy. The antimicrobial resistance profiles of methicillin-susceptible S. aureus (MSSA) isolates are also reported, together with the genotypic characteristics of mecA and mecCpositive MRSA strains.

\section{MATERIALS AND METHODS}

\section{Sample Collection}

Between January and June 2012, a total of 286 BTM samples were collected from 286 dairy sheep farms located in central Italy (Lazio region). The milk samples, collected by trained technicians, were transported to the laboratory in ice-cooled containers and analyzed within 24 to $48 \mathrm{~h}$ after collection.

\section{S. aureus Isolation and Identification}

All collected samples were analyzed for the enumeration of coagulase-positive staphylococci using BairdParker agar with rabbit plasma fibrinogen supplement according to ISO 6888-2: 1999 and Amd1: 2003 (ISO, 2003). Coagulase-positive colonies were identified as Staphylococcus spp. by microscopic observation, Gram staining, and catalase determination. Considering the composite nature of BTM samples, multiple suspected colonies (up to 5) were further analyzed from each positive sample. Genomic DNA was obtained from Staphylococcus spp. colonies previously subcultured on blood agar (5\% defibrinated bovine blood) by using InstaGene Matrix (Bio-Rad, Milano, Italy), as reported by Bianchi et al. (2014). Staphylococcus aureus identification was performed by a modified species-specific PCR, using primers targeting the fem $A$ gene (Mehrotra et al., 2000).

\section{Screening for Methicillin Resistance by Cefoxitin Disk Diffusion Test}

A total of 679 S. aureus colonies were screened for methicillin resistance by the cefoxitin disk diffusion test according to the criteria of Clinical Laboratory Standards Institute (CLSI). The results were interpreted following the Performance Standards for Antimicrobial Susceptibility Testing, Twenty-Third Informational Supplement (CLSI, 2013a).

\section{Molecular Characterization}

Cefoxitin-resistant isolates were tested for the presence of the mecA/mecC and blaZ genes by PCR assays using primers and protocols described by Stegger et al. (2012) and Martineau et al. (2000), respectively. The MRSA isolates were further genotyped by spa typing, multilocus sequence typing (MLST) and by typing/ subtyping of the staphylococcal cassette chromosome mec (SCCmec) using multiplex PCR methods as previously described (Battisti et al., 2010; Shore et al., 2011). The mec $C$-positive isolates were also tested by PCR analysis for the presence of the bla $Z$ allotype associated with SCCmec XI (blaZ-SCCmecXI), as reported by García-Álvarez et al. (2011).

The mecA/mec $C$-positive isolates were also screened by PCR analysis for the presence of specific immune evasion and virulence genes. These included the genes coding for the Panton-Valentine leucocidin (PVL) and 
the toxic shock syndrome toxin 1 (TSST-1; Fueyo et al., 2005), the immune evasion cluster (IEC) genes $s a k$ (staphylokinase) and scn (staphylococcal complement inhibitor precursor), as reported by van Wamel et al. (2006). The presence of 9 selected $S$. aureus enterotoxins (sea, seb, sec, sed, see, seg, seh, sei, and ser) and 2 staphylococcal-like enterotoxin $($ selj, selp) genes was assessed in the 2 MRSA isolates by using 2 multiplex PCR protocols, as previously described (Kérouanton et al., 2007; Bianchi et al., 2014).

\section{Antimicrobial Susceptibility Testing}

Selected MSSA isolates were tested for phenotypic susceptibility to antimicrobials representative of the most relevant classes active against Staphylococcus spp. by using the Kirby-Bauer disk diffusion method (CLSI, 2013a,b). Isolates selection was performed by choosing one single isolate from each positive sample/farm, when still available/vital. The results were interpreted according to the criteria of CLSI (2013a,b). The following antimicrobials were tested: ampicillin, amoxicillin/ clavulanic acid, cefotaxime, tetracycline, sulfonamides, chloramphenicol, gentamicin, trimethoprim/sulfamethoxazole, clindamycin, enrofloxacin, erythromycin, cephalothin, and kanamycin.

The mec-positive isolates were also tested for their antimicrobial susceptibility by the broth microdilution method (Trek Diagnostic Systems, Westlake, OH). The following drugs were tested: penicillin, cefoxitin, ciprofloxacin, chloramphenicol, clindamycin, erythromycin, gentamicin, kanamycin, streptomycin, linezolid, quinopristin/dalfopristin, fusidic acid, mupirocin, rifampicin, tetracycline, tiamulin, sulfamethoxazole, trimethoprim, and vancomycin. Minimum inhibitory concentrations were determined, and results were interpreted according to the European Committee on Antimicrobial Susceptibility Testing (EUCAST; http://www.eucast.org), using epidemiological cut-offs for the categorization of "microbiological resistance" or "non-wild-type" isolates. Results for the quality control documents were within published ranges.

\section{RESULTS}

\section{S. aureus and MRSA Detection}

Staphylococcus aureus was detected in 153 out of 286 BTM samples tested (53.5\%, 95\% CI: 47.54-59.37). The average $S$. aureus count was $2.53 \log \mathrm{cfu} / \mathrm{mL}$, with counts ranging from 1.26 to $4.63 \mathrm{log} \mathrm{cfu} / \mathrm{mL}$. Two out of the $679 \mathrm{~S}$. aureus colonies screened were resistant to cefoxitin. These isolates were from 2 samples collected in 2 different farms and carried the mecA/blaZ and mec $C$ genes, respectively. Both MRSA-positive farms consisted of semi-extensive dairy sheep herds with an average of 500 lactating ewes mechanically milked and no other livestock animals reared. The overall estimated between-herd MRSA prevalence was $0.70 \%(2 / 286$, 95\% CI: $0.12-2.78)$.

\section{Characterization of the MRSA Isolates}

The mecA-positive isolate belonged to spa type t127, Sequence Type (ST) (CC)1, harbored a SCCmec type IVa, carried also the blaZ gene encoding for $\beta$-lactamase, and was phenotypically resistant to all the $\beta$-lactams tested, and to erythromycin, streptomycin, kanamycin, and tetracycline. The isolate was detected in the same holding where previous investigations had already reported the presence of similar spa type t127, ST1, mecA-positive MRSA (Carfora et al., 2016). The mec $C$-positive isolate belonged to spa type t843, $\mathrm{ST}(\mathrm{CC}) 130$, harbored a SCCmec type XI, was negative for the chromosomally or plasmid-associated blaZ gene with primers and conditions as described by Martineau et al. (2000), and was positive, as expected, to the blaZ allotype associated with SCCmec XI (blaZ-SCCmecXI). It was phenotypically resistant to all the $\beta$-lactams, but susceptible to all the other antimicrobials tested. Both ST1 and ST130 MRSA were negative for the genes coding PVL, TSST-1, and IEC. Regarding the presence of the major S. aureus enterotoxin genes, the ST130 mecC-positive MRSA tested negative, whereas the ST1 mecA-positive MRSA harbored the seh gene, as expected (Alba et al., 2015).

\section{Characterization of the MSSA Isolates}

Among the 104 MSSA isolates examined for antimicrobial susceptibility, $63(60.58 \%)$ were susceptible to all the 13 antimicrobials tested, and $41(39.42 \%)$ were resistant to at least 1 antimicrobial. The resistance profiles of the MSSA are reported in Table 1. Overall, 22 isolates $(21.15 \%)$ were resistant to 1 single antimicrobial drug, 16 (15.38\%) to 2 drugs, whereas a multidrug resistance pattern (resistance to at least 3, and up to 4 antimicrobial drugs) was observed in 3 isolates $(2.88 \%)$. In particular, 23 isolates $(22.12 \%)$ were resistant to tetracycline, $16(15.38 \%)$ to sulfonomides, $14(13.46 \%)$ to trimethoprim and sulfamethoxazole, and $9(8.65 \%)$ to ampicillin. One isolate showed resistance to both enrofloxacin and kanamycin. None of the 104 MSSA isolates showed resistance to amoxicillin-clavulanic acid, cefotaxime, cephalothin, chloramphenicol, gentamicin, clindamycin, and erythromycin. 


\section{DISCUSSION}

Although S. aureus is frequently isolated from sheep raw milk and dairy products (Morandi et al., 2009; Spanu et al., 2014; Carfora et al., 2015; Jamali et al., 2015), the detection of MRSA in ovine milk (ArizaMiguel et al., 2014; Caruso et al., 2015; Pexara et al., 2015; Carfora et al., 2016) and derived dairy products (Normanno et al., 2007; Shanehbandi et al., 2014; Carfora et al., 2015) is sporadic.

In this survey, conducted in dairy sheep farms located in central Italy, we found a high prevalence of $S$. aureus contamination in ovine BTM (53.5\% of samples tested). Other studies conducted on BTM in different countries have reported lower prevalence rates, ranging from 9.6 to 33.3\% (Muehlherr et al., 2003; Scherrer et al., 2004; de Garnica et al., 2013; Pexara et al., 2015; Zdragas et al., 2015).

Despite this high overall $S$. aureus prevalence, the estimated MRSA prevalence was low $(0.7 \% ; 2 / 286)$. This finding is in agreement with those of other recent investigations carried out on small ruminant BTM in Italy (Caruso et al., 2015; Cortimiglia et al., 2015), Spain (Ariza-Miguel et al., 2014), and Greece (Pexara et al., 2015; Zdragas et al., 2015), which reported prevalence rates ranging from 0 to $2 \%$.

Interestingly, 1 of the 2 MRSA isolates identified carried a mecC gene. The presence of $m e c C$-positive MRSA has been already reported in ruminant farms in different European countries (García-Álvarez et al., 2011; Eriksson et al., 2013; Petersen et al., 2013; Unnerstad et al., 2013; Ariza-Miguel et al., 2014; Loncaric et al., 2014; Paterson et al., 2014), meaning a wide geographical spread of these strains. However, to date, $S$. aureus carrying the mecC gene have never been isolated from humans or animals in Italy. Similarly to $m e c A$-MRSA lineages, mecC-MRSA strains are highly versatile pathogens able to cause a wide range of infec-

Table 1. Antimicrobial resistance profiles of the methicillin-susceptible Staphylococcus aureus (MSSA) isolated from sheep bulk tank milk samples tested by the disk diffusion method $^{1}$

\begin{tabular}{lc}
\hline Resistance profile $^{2}$ & No. of resistant isolates \\
\hline TE & 16 \\
AM & 6 \\
TE-AM & 2 \\
TE-SUL & 2 \\
SUL-SXT & 12 \\
TE-SUL-SXT & 2 \\
TE-ENR-AM-K & 1 \\
Total & 41 \\
\hline
\end{tabular}

${ }^{1}$ Forty-one out of $104 \mathrm{MSSA}$ isolates tested were resistant to at least 1 antimicrobial.

${ }^{2} \mathrm{TE}=$ tetracycline; $\mathrm{AM}=$ ampicillin; $\mathrm{SUL}=$ sulfonamides; $\mathrm{SXT}=$ trimethoprim-sulfamethoxazole; ENR = enrofloxacin, $\mathrm{K}=$ kanamycin. tions in several host species (Paterson et al., 2014). The $M e c C$-MRSA have been regarded as animal-adapted lineages, probably arisen in animals, possibly ruminants, and subsequently spread to humans (Harrison et al., 2013; Petersen et al., 2013). In this regard, although it was detected in only 1 of the 286 farms tested, the isolation of a mec $C$-positive strain from an ovine BTM sample is of public health relevance because of its zoonotic potential (Harrison et al., 2013; Petersen et al., 2013). The MLST analysis revealed that the mecC isolate belonged to spa type t843, ST(CC)130, SCCmec type XI, which is a common lineage of mecC-MRSA reported in cattle and sheep in Europe, and is capable of causing infections in humans (García-Álvarez et al., 2011; Eriksson et al., 2013; Petersen et al., 2013; ArizaMiguel et al., 2014; Loncaric et al., 2014; Kerschner et al., 2015). As expected, our ST130 mecC-positive isolate was negative for the presence of specific phageborne virulence and immune evasion genes such as PVL, TSST-1, and IEC, often harbored by certain human-adapted community-acquired (CA) MRSA lineages. This finding is consistent with an animal-host origin of this clone (Cuny et al., 2011; Paterson et al., 2014). As for the antimicrobial resistance, whereas the $\mathrm{ST}(\mathrm{CC}) 1$ mecA-positive isolate was also resistant to tetracycline, erythromycin, streptomycin, and kanamycin, the $\mathrm{ST}(\mathrm{CC}) 130$ mecC-positive isolate was resistant only to $\beta$-lactams, as already reported for other similar $m e c C$ isolates (Petersen et al., 2013; Ariza-Miguel et al., 2014; Loncaric et al., 2014). The observation that the ST130 mecC strain isolated in this study did not acquire further resistance determinants, suggests that this lineage may not have been extensively subjected to multiple antibacterial selection pressure (Ariza-Miguel et al., 2014).

As for the antimicrobial susceptibility testing of the MSSA isolates, we found that $60.58 \%$ of them were susceptible to all the drugs tested. Lower rates of antimicrobial resistance have been reported in other studies conducted in Greece (Pexara et al., 2015; Zdragas et al., 2015) and Brazil (Martins et al., 2015). The most common resistance was that to tetracycline, observed in $22.12 \%$ of the isolates, a rate higher than that described in other studies in Italy (Lollai et al., 2008), Turkey (Ünal et al., 2012), and Brazil (Martins et al., 2015), but lower than that reported in Iran by Jamali et al. (2015). A relatively high level of resistance to sulfonamides $(15.38 \%)$ and trimethoprim-sulfamethoxazole $(13.46 \%)$ was also observed, a result in contrast with other studies conducted in Iran (Jamali et al., 2015), Greece (Pexara et al., 2015; Zdragas et al., 2015), and Brazil (Martins et al., 2015), reporting resistance rates ranging from 0 to $1.2 \%$. On the other hand, the ampicillin resistance rate $(8.65 \%)$ was similar to those 
reported in other studies conducted in Slovenia (Pengov and Ceru, 2003) and Greece (Zdragas et al., 2015). In agreement with other studies conducted in Italy (Spanu et al., 2014), Greece (Pexara et al., 2015), and Brazil (Martins et al., 2015), multidrug resistance $(\geq 3$ antimicrobial drugs) was observed only in a small proportion of our MSSA isolates (2.88\%), supporting the view that it still represents an occasional occurrence in MSSA of ovine origin (Spanu et al., 2014; Pexara et al., 2015). The relatively low rate of multi-drug resistance observed could be probably ascribed to the extensive or semi-extensive farming systems often associated with sheep breeding, and the consequent limited use of antimicrobials in this species, as compared with other intensive farming ones.

In conclusion, this study represents the first report of mecC-positive MRSA isolation in Italy and would confirm that, besides CC1-mecA-positive-LA-MRSA, sheep might act as a reservoir of $S$. aureus clones, such as CC130, prone to harbor mec $C$ genes. In this regard, it should be considered that the prevalence of mecCpositive LA-MRSA could be underestimated because they are difficult to identify by diagnostic methods routinely employed for the detection of mecA-mediated methicillin resistance. Indeed, mec $C$-positive MRSA show low-level oxacillin resistance with negative PBP2a detection, if not tested after induction by cefoxitin, and are not detected by mecA PCR (García-Álvarez et al., 2011; Deplano et al., 2014). In addition, it has been reported that MRSA CC130 strains grow insufficiently on commercial selective chromogenic agar plates for the detection of MRSA (Cuny et al., 2011). The high prevalence of $S$. aureus found in BTM samples and the isolation of MRSA, although at a low prevalence, underlines the importance of adopting control measures against $S$. aureus in dairy sheep farms to minimize the risks for both animal and public health. In this regard, the implementation of specific monitoring/surveillance programs at the national or regional level (or both) would help in better understanding the epidemiology and trends of LA-MRSA in dairy small ruminant herds, along with specific studies aimed at investigating the risk factors involved in sheep and goat colonization/ infection and between-herd transmission.

\section{ACKNOWLEDGMENTS}

The authors thank Adreana Tammaro and Emanuela Bovi, National Reference Centre for Ovine and Caprine Milk and Dairy Products Quality (CRELDOC), Istituto Zooprofilattico Sperimentale del Lazio e della Toscana; and Carmela Buccella, Gessica Cordaro, and Paola di Matteo, General Diagnostic Department, National Reference Laboratory for Antimicrobial Resistance,
Istituto Zooprofilattico Sperimentale del Lazio e della Toscana, for their outstanding technical assistance. This work was supported by the Italian Ministry of Health (Ricerca Corrente IZSLT 11/11). We declare no conflicts of interest for this work.

\section{REFERENCES}

Alba, P., F. Feltrin, G. Cordaro, M. C. Porrero, B. Kraushaar, M. A. Argudín, S. Nykäsenoja, M. Monaco, M. Stegger, F. M. Aarestrup, P. Butaye, A. Franco, and A. Battisti. 2015. Livestock-associated methicillin resistant and methicillin susceptible Staphylococcus aureus sequence type (CC) 1 in European farmed animals: High genetic relatedness of isolates from Italian cattle herds and humans. PLoS One 10:e0137143. https://doi.org/10.1371/journal pone.0137143.

Angen, Ø., M. Stegger, J. Larsen, B. Lilje, H. Kaya, K. S. Pedersen, A. Jakobsen, A. Petersen, and A. R. Larsen. 2017. Report of mecCcarrying MRSA in domestic swine. J. Antimicrob. Chemother. 72:60-63.

Ariza-Miguel, J., M. Hernandez, I. Fernandez-Natal, and D. RodriguezLazaro. 2014. Methicillin-resistant Staphylococcus aureus harboring mecC in livestock in Spain. J. Clin. Microbiol. 52:4067-4069.

Battisti, A., A. Franco, G. Merialdi, H. Hasman, M. Iurescia, R. Lorenzetti, F. Feltrin, M. Zini, and F. M. Aarestrup. 2010. Heterogeneity among methicillin-resistant Staphylococcus aureus from Italian pig finishing holdings. Vet. Microbiol. 142:361-366. https://doi .org/10.1016/j.vetmic.2009.10.008.

Bergonier, D., R. de Crémoux, R. Rupp, G. Lagriffoul, and X. Berthelot. 2003. Mastitis of dairy small ruminants. Vet. Res. 34:689-716.

Bianchi, D. M., S. Gallina, A. Bellio, F. Chiesa, T. Civera, and L. Decastelli. 2014. Enterotoxin gene profiles of Staphylococcus aureus isolated from milk and dairy products in Italy. Lett. Appl. Microbiol. 58:190-196. https://doi.org/10.1111/lam.12182.

Carfora, V., A. Caprioli, N. Marri, D. Sagrafoli, C. Boselli, G. Giacinti, G. Giangolini, L. Sorbara, S. Dottarelli, A. Battisti, and S. Amatiste. 2015. Enterotoxin genes, enterotoxin production, and methicillin resistance in Staphylococcus aureus isolated from milk and dairy products in Central Italy. Int. Dairy J. 42:12-15. https://doi .org/10.1016/j.idairyj.2014.10.009.

Carfora, V., G. Giacinti, D. Sagrafoli, N. Marri, G. Giangolini, P. Alba, F. Feltrin, L. Sorbara, R. Amoruso, A. Caprioli, S. Amatiste, and A. Battisti. 2016. Methicillin-resistant and methicillin-susceptible Staphylococcus aureus in dairy sheep and in-contact humans: An intra-farm study. J. Dairy Sci. 99:4251-4258. https://doi.org/ 10.3168/jds.2016-10912.

Caruso, M., L. Latorre, G. Santagada, R. Fraccalvieri, A. Miccolupo, R. Sottili, L. Palazzo, and A. Parisi. 2015. Methicillin-resistant Staphylococcus aureus (MRSA) in sheep and goat bulk tank milk from Southern Italy. Small Rumin. Res. 135:26-31. https://doi .org/10.1016/j.smallrumres.2015.12.023.

Chua, K. Y., B. P. Howden, J. H. Jiang, T. Stinear, and A. Y. Peleg. 2014. Population genetics and the evolution of virulence in Staphylococcus aureus. Infect. Genet. Evol. 21:554-562. https://doi.org/ 10.1016/j.meegid.2013.04.026.

Clinical and Laboratory Standard Institute (CLSI). 2013a. M02-A11 AND M100-S23. Performance Standards for Antimicrobial Disk Susceptibility Tests; Approved Standard-Eleventh Edition \& Performance Standards for Antimicrobial Susceptibility Testing; Twenty-Third Informational Supplement. CLSI, Wayne, PA.

Clinical and Laboratory Standard Institute (CLSI). 2013b. VET01-A4 and VET01-S2-Performance Standards for Antimicrobial Disk and Dilution Susceptibility Tests for Bacterial Isolated from Animals; Approved Standard-Fourth Edition and Supplement, VET01A4E and VET01S2E. CLSI, Wayne, PA.

Cortimiglia, C., V. Bianchini, A. Franco, A. Caprioli, A. Battisti, L. Colombo, K. Stradiotto, F. Vezzoli, and M. Luini. 2015. Short communication: Prevalence of Staphylococcus aureus and methicillin-resistant $S$. aureus in bulk tank milk from dairy goat farms 
in northern Italy. J. Dairy Sci. 98:2307-2311. https://doi.org/10 $.3168 /$ jds.2014-8923.

Cuny, C., F. Layer, B. Strommenger, and W. Witte. 2011. Rare occurrence of methicillin-resistant Staphylococcus aureus CC130 with a novel mecA homologue in humans in Germany. PLoS One 6:e24360. https://doi.org/10.1371/journal.pone.0024360.

de Garnica, M. L., B. Linage, J. A. Carriedo, J. A. Santos, and C. Gonzalo. 2013. Staphylococcus aureus and Escherichia coli prevalence in ovine bulk tank milk. Small Rumin. Res. 115:108-112.

Deplano, A., S. Vandendriessche, C. Nonhoff, and O. Denis. 2014. Genetic diversity among methicillin-resistant Staphylococcus aureus isolates carrying the mec $\mathrm{C}$ gene in Belgium. J. Antimicrob. Chemother. 69:1457-1460.

Eriksson, J., C. Espinosa-Gongora, I. Stamphøj, A. R. Larsen, and L. Guardabassi. 2013. Carriage frequency, diversity and methicillin resistance of Staphylococcus aureus in Danish small ruminants. Vet. Microbiol. 163:110-115. https://doi.org/10.1016/j.vetmic .2012 .12 .006

Feltrin, F., P. Alba, B. Kraushaar, A. Ianzano, M. A. Argudín, P. Di Matteo, M. C. Porrero, F. M. Aarestrup, P. Butaye, A. Franco, and A. Battisti. 2015. Molecular characterization of a livestockassociated, multidrug-resistant, methicillin-resistant Staphylococcus aureus Clonal Complex 97 lineage spreading in dairy cattle and pigs in Italy. Appl. Environ. Microbiol. 82:816-821. https:// doi.org/10.1128/AEM.02854-15.

Fessler, A. T., R. G. Olde Riekerink, A. Rothkamp, K. Kadlec, O. C. Sampimon, T. J. Lam, and S. Schwarz. 2012. Characterization of methicillin-resistant Staphylococcus aureus CC398 obtained from humans and animals on dairy farms. Vet. Microbiol. 160:77-84. https://doi.org/10.1016/j.vetmic.2012.05.005.

Fueyo, J. M., M. C. Mendoza, M. R. Rodicio, J. Muñiz, M. A. Alvarez, and M. C. Martín. 2005. Cytotoxin and pyrogenic toxin superantigen gene profiles of Staphylococcus aureus associated with subclinical mastitis in dairy cows and relationships with macrorestriction genomic profiles. J. Clin. Microbiol. 43:1278-1284.

García-Álvarez, L., M. T. G. Holden, H. Lindsay, C. R. Webb, D. F. Brown, M. D. Curran, E. Walpole, K. Brooks, D. J. Pickard, C. Teale, J. Parkhill, S. D. Bentley, G. F. Edwards, E. K. Girvan, A. M. Kearns, B. Pichon, R. L. Hill, A. R. Larsen, R. L. Skov, S. J. Peacock, D. J. Maskell, and M. A. Holmes. 2011. Meticillinresistant Staphylococcus aureus with a novel mecA homologue in human and bovine populations in the UK and Denmark: A descriptive study. Lancet Infect. Dis. 11:595-603.

Guardabassi, L., J. Larsen, J. S. Weese, P. Butaye, A. Battisti, J. Kluytmans, D. H. Lloyd, and R. L. Skov. 2013. Public health impact and antimicrobial selection of methicillin-resistant staphylococci in animals. J. Glob. Antimicrob. Resist. 1:55-62.

Hanselman, B. A., S. A. Kruth, J. Rousseau, D. E. Low, B. M. Willey, A. McGeer, and J. S. Weese. 2006. Methicillin resistant Staphylococcus aureus colonization in veterinary personnel. Emerg. Infect. Dis. 12:1933-1938.

Harrison, E. M., G. K. Paterson, M. T. Holden, J. Larsen, M. Stegger, A. R. Larsen, A. Petersen, R. L. Skov, J. M. Christensen, A. Bak Zeuthen, O. Heltberg, S. R. Harris, R. N. Zadoks, J. Parkhill, S. J. Peacock, and M. A. Holmes. 2013. Whole genome sequencing identifies zoonotic transmission of MRSA isolates with the novel $m e c \mathrm{~A}$ homologue mecC. EMBO Mol. Med. 5:509-515.

ISO. 2003. 6888-2: 1999/Amd1: Microbiology of food and animal feeding stuff-Horizontal method for the enumeration of coagulasepositive staphylococci (Staphylococcus aureus and other species)Part 2: Technique using rabbit plasma fibrinogen agar medium and amendment 1: Inclusion of precision data. International Organization for Standardisation, Geneva, Switzerland.

Jamali, H., M. Paydar, B. Radmehr, S. Ismail, and A. Dadrasnia. 2015. Prevalence and antimicrobial resistance of Staphylococcus aureus isolated from raw milk and dairy products. Food Contr. 54:383-388.

Kérouanton, A., J. A. Hennekinne, C. Letertre, L. Petit, O. Chesneau, A. Brisabois, and M. L. De Buyser. 2007. Characterization of Staphylococcus aureus strains associated with food poisoning outbreaks in France. Int. J. Food Microbiol. 115:369-375.
Kerschner, H., E. M. Harrison, R. Hartl, M. A. Holmes, and P. Apfalter. 2015. First report of $m e c \mathrm{C}$ MRSA in human samples from Austria: Molecular characteristics and clinical data. New Microbes and New Infections 3:4-9. https://doi.org/10.1016/j.nmni.2014.11 .001 .

Kluytmans, J. A. 2010. Methicillin-resistant Staphylococcus aureus in food products: Cause for concern or case for complacency? Clin. Microbiol. Infect. 16:11-15. https://doi.org/10.1111/j.1469-0691 2009.03110.x

Lollai, S. A., M. Ziccheddu, C. Di Mauro, D. Manunta, A. Nudda, and G. Leori. 2008. Profile and evolution of antimicrobial resistance of ovine mastitis pathogens (1995-2004). Small Rumin. Res. $74: 249-254$.

Loncaric, I., A. Kubber-Heiss, A. Posautz, G. L. Stalder, D. Hoffmann, R. Rosengarten, and C. Walter. 2014. mecC- and mecA-positive meticillin-resistant Staphylococcus aureus (MRSA) isolated from livestock sharing habitat with wildlife previously tested positive for mecC-positive MRSA. Vet. Dermatol. 25:147-148. https://doi .org/10.1111/vde.12116.

Luini, M., P. Cremonesi, G. Magro, V. Bianchini, G. Minozzi, B. Castiglioni, and R. Piccinini. 2015. Methicillin-resistant Staphylococcus aureus (MRSA) is associated with low within-herd prevalence of intra-mammary infections in dairy cows: Genotyping of isolates. Vet. Microbiol. 178:270-274. https://doi.org/10.1016/j.vetmic 2015.05.010.

Martineau, F., F. J. Picard, L. Grenier, P. H. Roy, M. Ouellette, and M. G. Bergeron. 2000. Multiplex PCR assays for the detection of clinically relevant antibiotic resistance genes in staphylococci isolated from patients infected after cardiac surgery. The ESPRIT trial. J. Antimicrob. Chemother. 46:527-534.

Martins, K. B., P. Y. Faccioli-Martins, D. F. Riboli, V. C. Pereira S. Fernandes, A. A. Oliveira, A. Dantas, L. F. Zafalon, and L. da Cunha Mde. 2015. Clonal profile, virulence and resistance of Staphylococcus aureus isolated from sheep milk. Braz. J. Microbiol. 46:535-543. https://doi.org/10.1590/S1517-838246220131164.

Mehrotra, M., G. Wang, and W. M. Johnson. 2000. Multiplex PCR for detection of genes for Staphylococcus aureus enterotoxins, exfoliative toxins, toxic shock syndrome toxin 1 , and methicillin resistance. J. Clin. Microbiol. 38:1032-1035.

Morandi, S., M. Brasca, C. Andrighetto, A. Lombardi, and R. Lodi. 2009. Phenotypic and genotypic characterization of Staphylococcus aureus strains from Italian dairy products. Int. J. Microbiol https://doi.org/10.1155/2009/501362.

Muehlherr, J. E., C. Zweifel, S. Corti, J. E. Blanco, and R. Stephan. 2003. Microbiological quality of bulk-tank raw milk of goat and ewe in Switzerland. J. Dairy Sci. 86:3849-3856.

Normanno, G., M. Corrente, G. La Salandra, A. Dambrosio, N. C. Quaglia, A. Parisi, G. Greco, A. L. Bellacicco, S. Virgilio, and G. V. Celano. 2007. Methicillin-resistant Staphylococcus aureus (MRSA) in foods of animal origin product in Italy. Int. J. Food Microbiol. 117:219-222. https://doi.org/10.1016/j.ijfoodmicro 2007.04.006

Paterson, G. K., E. M. Harrison, and M. A. Holmes. 2014. The emergence of mecC methicillin-resistant Staphylococcus aureus. Trends Microbiol. 22:42-47.

Pengov, A., and S. Ceru. 2003. Antimicrobial drug susceptibility of Staphylococcus aureus strains isolated from bovine and ovine mammary glands. J. Dairy Sci. 86:3157-3163.

Petersen, A., M. Stegger, O. Heltberg, J. Christensen, A. Zeuthen, L. K. Knudsen, T. Urth, M. Sorum, L. Schouls, J. Larsen, R. Skov, and A. R. Larsen. 2013. Epidemiology of methicillin-resistant Staphylococcus aureus carrying the novel mecC gene in Denmark corroborates a zoonotic reservoir with transmission to humans. Clin. Microbiol. Infect. 19:E16-E22. https://doi.org/10.1111/1469 $-0691.12036$

Pexara, A., N. Solomakos, D. Sergelidis, A. S. Angelidis, and A. Govaris. 2015. Occurrence and antibiotic resistance of enterotoxigenic Staphylococcus aureus in raw ovine and caprine milk in Greece. Dairy Sci. Technol. https://doi.org/10.1007/s13594-015-0272-z.

Scherrer, D., S. Corti, J. E. Muehlherr, C. Zweifel, and R. Stephan. 2004. Phenotypic and genotypic characteristics of Staphylococ- 
cus aureus isolates from raw bulk-tank milk samples of goats and sheep. Vet. Microbiol. 101:101-107.

Shanehbandi, D., B. Baradaran, S. Sadigh-Eteghad, and H. Zarredar. 2014. Occurrence of methicillin resistant and enterotoxigenic Staphylococcus aureus in traditional cheeses in the north west of Iran. ISRN Microbiol. https://doi.org/10.1155/2014/129580.

Shore, A. C., E. C. Deasy, P. Slickers, G. Brennan, B. O'Connell, S. Monecke, R. Ehricht, and D. C. Coleman. 2011. Detection of staphylococcal cassette chromosome mec type XI carrying highly divergent mecA, mecI, mecR1, blaZ, and ccr genes in human clinical isolates of clonal complex 130 methicillin-resistant Staphylococcus aureus. Antimicrob. Agents Chemother. 55:3765-3773.

Spanu, V., C. Scarano, F. Cossu, C. Pala, C. Spanu, and E. P. De Santis. 2014. Antibiotic resistance traits and molecular subtyping of Staphylococcus aureus isolated from raw sheep milk cheese. J. Food Sci. 79:M2066-M2071.

Stegger, M., P. S. Andersen, A. Kearns, B. Pichon, M. A. Holmes, G. Edwards, F. Laurent, C. Teale, R. Skov, and A. R. Larsen. 2012. Rapid detection, differentiation and typing of methicillin-resistant Staphylococcus aureus harboring either mecA or the new mecA homologue mecALGA251. Clin. Microbiol. Infect. 18:395-400. https://doi.org/10.1111/j.1469-0691.2011.03715.x.

Ünal,, N., S. Askar, H. C. Macun, F. Sakarya, B. Altun, and M. Ylldırım. 2012. Panton-Valentine leukocidin and some exotoxins of Staphylococcus aureus and antibiotic susceptibility profiles of staphylococci isolated from milk of small ruminants. Trop. Anim. Health Prod. 44:573-579.

Unnerstad, H. E., B. Bengtsson, M. Horn af Rantzien, and S. Börjesson. 2013. Methicillin-resistant Staphylococcus aureus containing $m e c \mathrm{C}$ in Swedish dairy cows. Acta Vet. Scand. 55:6. https://doi .org/10.1186/1751-0147-55-6.

van Wamel, W. J., S. H. Rooijakkers, M. Ruyken, K. P. van Kessel, and J. A. van Strijp. 2006. The innate immune modulators staphylococcal complement inhibitor and chemotaxis inhibitory protein of Staphylococcus aureus are located on beta-hemolysin-converting bacteriophages. J. Bacteriol. 188:1310-1315.

Vanderhaeghen, W., K. Hermans, F. Haesebrouck, and P. Butaye. 2010. Methicillin resistant Staphylococcus aureus (MRSA) in food production animals. Epidemiol. Infect. 138:606-625.

Zdragas, A., T. Papadopoulos, I. Mitsopoulos, G. Samouris, G. Vafeas, E. Boukouvala, L. Ekateriniadou, K. Mazaraki, A. Alexopoulos, and V. Lagka. 2015. Prevalence, genetic diversity, and antimicrobial susceptibility profiles of Staphylococcus aureus isolated from bulk tank milk from Greek traditional ovine farms. Small Rumin. Res. 125:120-126. 